\title{
Evaluation of Nestin and EGFR in Patients with Glioblastoma Multiforme in a Public Hospital in Iran
}

\author{
Amir Hassan Matini ${ }^{1}$, Mohadeseh Mofidi Naeini' ${ }^{1}$, Hamed Haddad Kashani², \\ Zarichehr Vakili ${ }^{1 *}$
}

\begin{abstract}
Introduction: Glioblastoma multiforme (GBM) is a grade IV glioma and accounts for 15\% of all primary brain tumors. This GBM has a median survival range of less than 2 years after diagnosis and it is highly vascularized by neoformed vessels. Neoangiogenesis is a crucial factor in the malignant tumoral behavior and prognosis of patients and Nestin protein belongs to class VI which is expressed in endothelial cells of neoformed vessels in GBM. Our study shows the correlation between EGFR mutation and Nestin expression in endothelial of neoformed vessels in GBM. Methods: We analyzed 40 GBM samples by immunohistochemistry staining. The immunohistochemical expression of EGFR in tumoral cells and Nestin in endothelial cells in paraffin sections were analyzed. EGFR scoring was the based on staining intensity. Score 0 shows No staining, Score1, mild to moderate staining and score2 sever staining. Microvascular density (MVD) was evaluated with Nestin-immunoreactive. Results: The mean of MVD was 14.6 \pm 8.25 . Nestin-MVD was significantly higher in GBM with sever vascular prolifration ( $\mathrm{p}$-value $=0.01$ ). EGFR was expressed in $92.5 \%$ of samples. The EGFR scoring for tumoral tissue was $7.5 \%($ score: 0 ), $22.5 \%$ (score:1) and $70 \%$ (score:2). There was a significant relationship between EGFR expression and MVD ( $\mathrm{p}$-value $=0.017$ ). Conclusion: We suggest that some important mutations as like as EGFR in GBM is responsible for inducing angiogenesis and vascular proliferation. Nestin overexpression as a novel marker might reflect the extent of neoangiogenesis, thus target therapy against EGFR pathway and anti angiogenic may be useful for GBM treatment.
\end{abstract}

Keywords: Nestin- angiogenesis- glioblastoma multiforme- microvascular density

Asian Pac J Cancer Prev, 21 (10), 2889-2894

\section{Introduction}

According to the American tumor association, brain tumors with prevalence of 12.8 per 100,000 are the most common tumors in the world and Gliomas are the most abundant. Gliomas are the brain parenchymal tumors which are similar in histology to different types of glial cells. The main types of gliomas are astrocytoma and oligodendrogliomas. Astrocytoma has different types that fibrillaryand pilocytic astrocytoma are the most common (Smith et al., 2012). World health organization (WHO) has divided these tumors in to 4 grades based on their cellularity, mitosis, necrosis and vascular proliferation (Pallini, Ricci-Vitiani et al., 2008; McNamara et al., 2013; Moghaddam et al., 2015). The most malignant astrocyte tumors is glioblastoma multiforme (GBM) (Batash et al., 2017). The survival of patients is approximately $12-15$ months and less than $3 \%$ of patients live more than 5 years(Pallini et al., 2008; McNamara et al., 2013). This tumor is common at age 45-50 years and including 9\% of children brain tumors (McNamara et al., 2013). GBM histologically is a heterogeneous tumor consisting of glioma tissue and numerous vessels (Schmidt et al., 2002; Tena-Suck et al., 2015). Although all glioblastoma are in one histological grade, the genetic changes are different (Simmons et al., 2001; Pallini et al., 2008; Liu et al., 2015). One of them is a mutation in Epidermal growth factor receptor (EGFR) gene (Nagpal et al., 2006; Lebelt et al., 2008; Liu et al., 2015). EGFR is a tyrosine kinase receptor which regulates cellular growth and differentiation. Amplifying $(40 \%<)$ and over expression $(60 \%>)$ of EGFR are considerable points in GBMs (Nagpal et al., 2006; Chinnaiyan et al., (2008); Lebelt et al., (2008). GBM has great ability in angiogenesis and proliferation and endothelial hyperplasia among the brain tumors. In fact, angiogenesis is a biological key and an important diagnostic marker for GBM (Ishiwata et al., 2011; Krupkova et al., 2011; Hardee and Zagzag 2012; Tena-Suck et al.. 2015). Angiogenesis is a complicated process in which endogenous markers, chemical signals and changes in the endothelial progenitor cells and surrounding stroma, stimulate and proliferate endothelial cells. In fact, angiogenesis has a key role in cancer growth and development (Kitai et al., 2010; Ishiwata et al., 2011). 
Angiogenesisin GBM may happen in response to the hypo oxidation in cells of tumor tissue. So, we can usually see proliferation around the necrotic areas(Ishiwata et al., 2011; Sasmita et al., 2018). But there are some evidences indicating that in addition to hypo oxidation, there are other mechanisms, such as mutation in p53, EGFR and growth factors independent to hypo oxidation such as VEGF genes that can lead to angiogenesis(Mokrý et al., 2004; Kitaiet al., 2010; Matsuda et al., 2013). According to the relation between angiogenesis and invasion andprognosis of GBM, study of angiogenesisusing vascular markers in neformed vessels can help with the diagnosis and prognosis of patients. Furthermore inhibition treatments against these markers, can prevent tumor angiogenesis and developments (Shih and Holland 2006; Veselska et al., 2006; Jin et al., 2013). Nestin is a class VI intermediate filaments that expresses in proliferative neuroepithelium during the embryonic development (Shih and Holland 2006; Loja et al., 2009; JR et al. 2010, Dahlrot, Hansen et al., 2014). In adults, Nestin is expressed in subventricular zone where neurogenesis occurs. In addition, Nestin expresses in growing endothelial progenitor cells but not in the matured endothelial cells. So Nestin expression is restricted to neoformed vessels and is more specific than the other markers (Shih and Holland, 2006). In fact, evaluation of the expression of this marker basedon the nuclear staining in immunohistochemistry (IHC) technique can lead to evaluation of micro vascular density (MVD) in tumor surface (Kitai et al., 2010; Ishiwata et al., 2011; Sica et al., 2011; Guadagno et al., 2016).

\section{Materials and Methos}

40 samples of GBM patients of Shahid Beheshti Hospital, Kashan, Iran were analyzed. The samples belonged to the years 2006-2016. Before selecting, the report on each sample and their H\&E slides were reviewed by pathologist. Then, 2 slides from each 5 micron paraffin section were made and stained by immunohistochemistry(IHC) staining using Nestin and EGFR markers. Micro vascular density (MVD) was evaluated by Nestin-immunoreactive.

Nestin protein expression was detected by Nestin antibody (10c2) (Santa Cruz Biotechnology, Inc.) as a yellow to brown color in the nucleus of vascular endothelial cells. Also the expression of EGFR in nucleus of tumoral cells was evaluated by observing yellow to brown color. In order to determine the Micro Vascular Density (MVD), at first, high density areas (Hot Spot) was determined in low magnification $(\times 40)$ and then they were counted in high magnification $(\times 400)$. Each endothelial cell relating to a hot spot was counted as a vessel and the mean of counted vessels in four fields were considered as the Nestin vascular density (Moghaddamet al., 2015). Anti-EGFR specially stains cell membrane and sometimes cytoplasm of cells. Scoring was based on the staining intensity. Score 0 was considered for no staining, score 1 for mild to moderate staining and Score 2 for severe staining.
Data were analyzed by SPSS version 17.0 software. Statistical tests such as chi-square and Fisher's exact test and odds ratio (OR) were used. P-Value was considered below $5 \%$.

\section{Ethical considerations}

The case study in this study is tissue sections. Each sample in the pathology laboratory has specific code and its sections has the same code. So at first, the samples were studied without any information. Then further information was gotten from the patients and analyzed. After making required slides, sections were returned to the laboratory.

\section{Results}

Necrosis and Vascular proliferation were observed as an important diagnostic marker in $90 \%$ of GBMs. Cellular atypia and pleomorphism were observed in all the tumors in which $17.5 \%$ showed mild atypia, $47.5 \%$ moderate atypia and 35\% severe atypia (Table 1).

Anti-EGFR staining as the EGFR mutation marker in malignant cells was negative in 3 patients and was positive in 37 patients. 8 patients showed score 1 and 29 patients showed score 2 . The frequency for score 0 was 3 (7.5\%), for score 1 was $9(22.5 \%)$ and for score 2 was 28 (70\%) (Figure 1). The mean of Micro Vascular Density

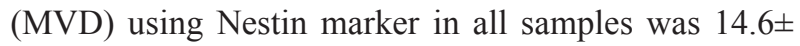
8.25 (Figure 2).

The mean of MVD in the group with vascular proliferation was $13.22 \pm 8.09$ and in the group without proliferation was $3.25 \pm 0.96$. The difference between two groups was significant $(\mathrm{P}$-value=0.01) (Figure3).

Frequency in the group with no EGFR expression (Figure 4) in which MVD was below themean, was 6, and with mild to moderate expression was 8 and with severe expression was 15 . Frequency in the group in which MVD was above the mean, just observing in severe EGFR expression, was 11. There was significant relation between MVD and EGFR expression and $\mathrm{P}_{-}$value was

Table 1. Pathological Features of Tumors

\begin{tabular}{lc}
\hline Pathologic features & Frequency \\
\hline Tumors with Necrosis & $36(90 \%)$ \\
Tumors without Necrosis & $4(10 \%)$ \\
Tumors with vascular proliferation & $36(90 \%)$ \\
Tumors without vascular proliferation & $4(10 \%)$ \\
Tumors with mild atypia and poleomorphism & $7(17.5 \%)$ \\
Tumors with moderate atypia and pleomorphism & $19(47.5 \%)$ \\
Tumors with severe atypia and pleomorphism & $14(35 \%)$ \\
\hline
\end{tabular}

Table 2. The Relation between EGFR Expression and MVD

\begin{tabular}{llccc}
\hline & & \multicolumn{3}{c}{ EGFR Expression } \\
& & Score 0 & Score 1 & Score 2 \\
\hline MVD & $\begin{array}{l}\text { Below the } \\
\text { mean }(<14.6) \\
\text { Above the } \\
\text { mean }(>14.6)\end{array}$ & $6(15 \%)$ & $8(20 \%)$ & $15(38 \%)$ \\
\hline
\end{tabular}




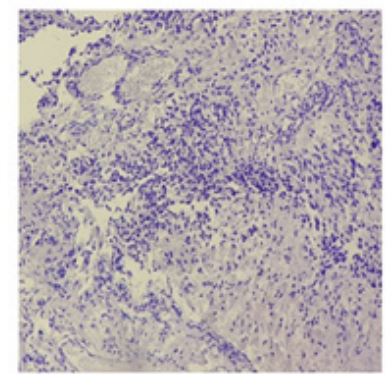

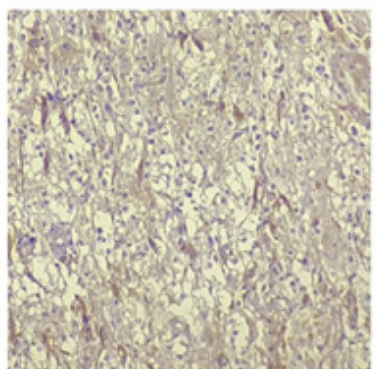

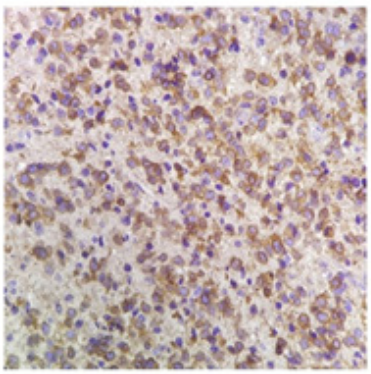

Figure 1. EGFR Marker Staining. A) Score 0, no staining; B) Score1, mild staining; C) Score2, severe staining

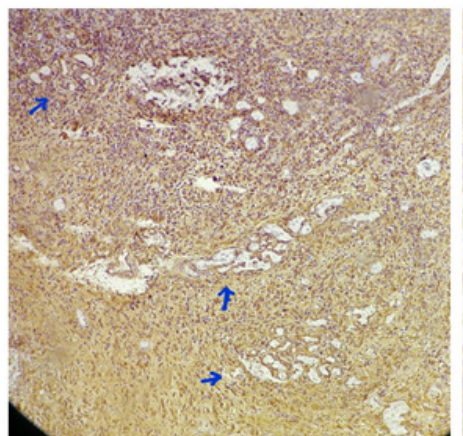

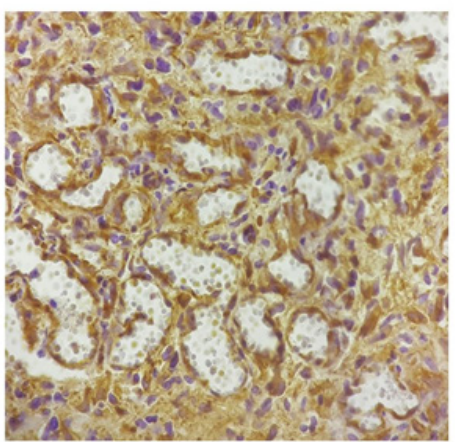

Figure 2. MVD Evaluating Using Nestin Marker. A) High density areas or Hot spots in low magnification $(\times 40)$. Each Blue arrow indicates a hotspot. B) High density areas in high magnification $(\times 400)$.

0.017 (Table 2). However, the MVD and Nestin showed an increased expression (Figure 5).

\section{Discussion}

Available treatments for GBM such as surgery, radiation and chemotherapy cannot survive the patients more than 14 months. So development of treatment approaches is an important issue (Cho et al., 2011). Although GBM is prevalent in patients of all ages, it is more observed in the elderly and in white men.

Jazayeri et al., (2013) reported that the mean age of GBM patients is $50.6 \pm 16.9$ in Iran and the incidents has been reported 0.79 case per 100,000 in years $2000-2010$
(Jazayeri et al., 2013).

In our study the mean age of patients was $53.32 \pm 16.04$ at the range of 18-88 years and it was more common in males. Necrosis, cellular atypia and vascular proliferation are the most important diagnostic factors in GBM.

Tena-Suck et al., (2015) have reported that necrosis was founded in $66 \%$ of primary tumors and $82 \%$ of secondary tumors. There were more vascular proliferation in more inflamed and necrotic tumors but there was no significant correlation (Tena-Suck et al., 2015).

In this study, necrosis was observed in $90 \%$ of samples but there was not any correlation between necrosis and age and vascular proliferation. However Cellular atypia and pleomorphism were found in all samples and were

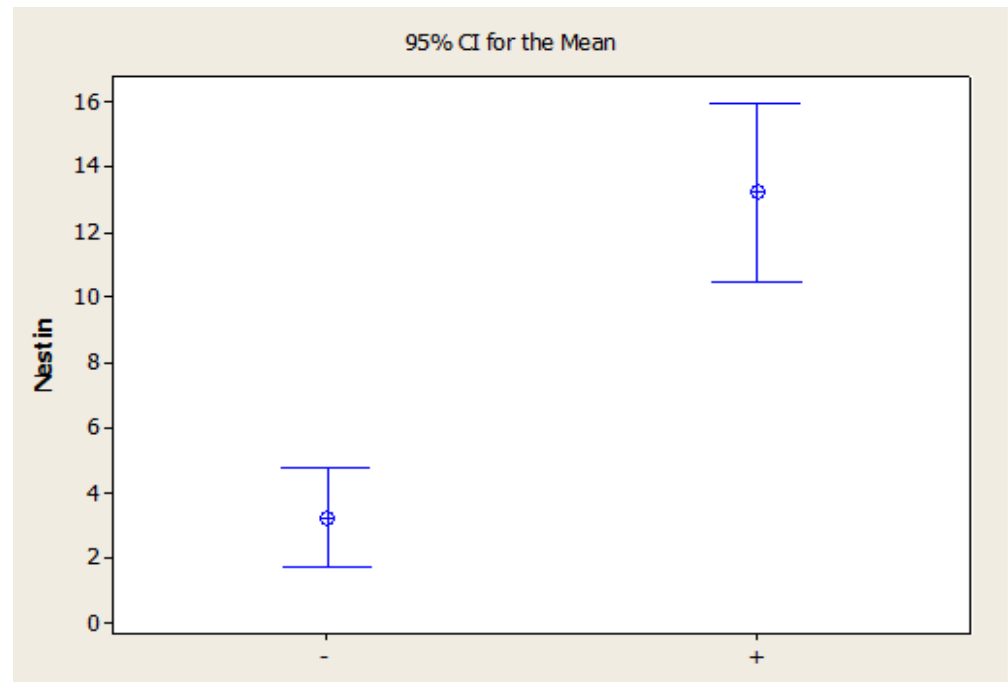

Figure 3. The Mean of Micro Vascular Density in Presence or Absence of Vascular Proliferation. 


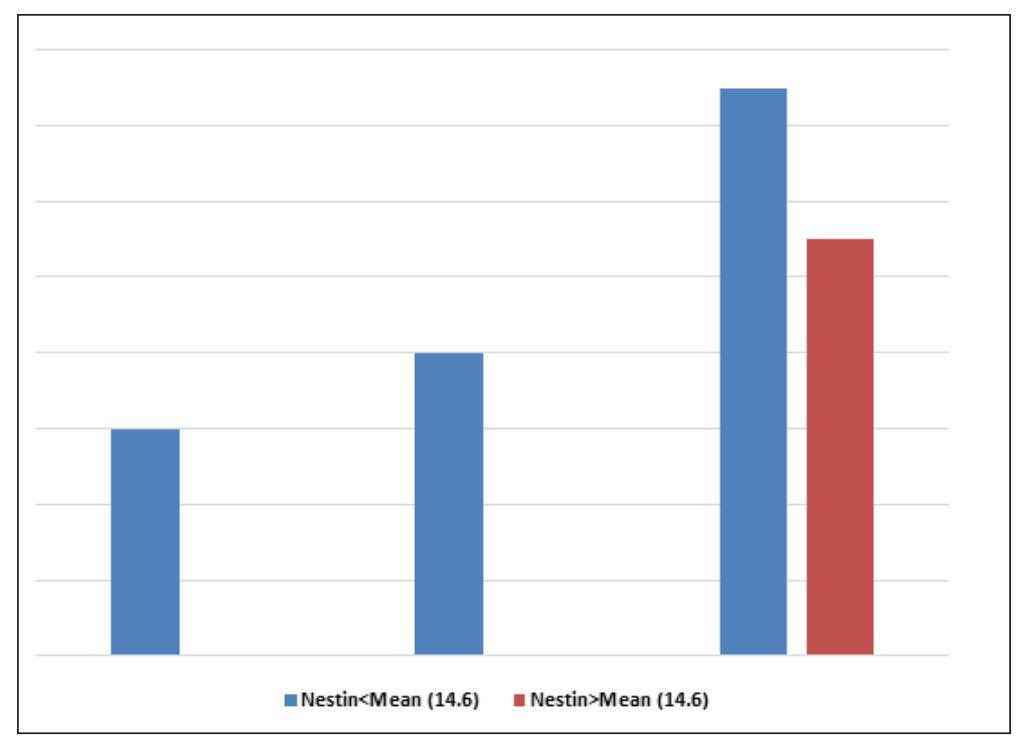

Figure 4. MVD Frequency and EGFR Expression

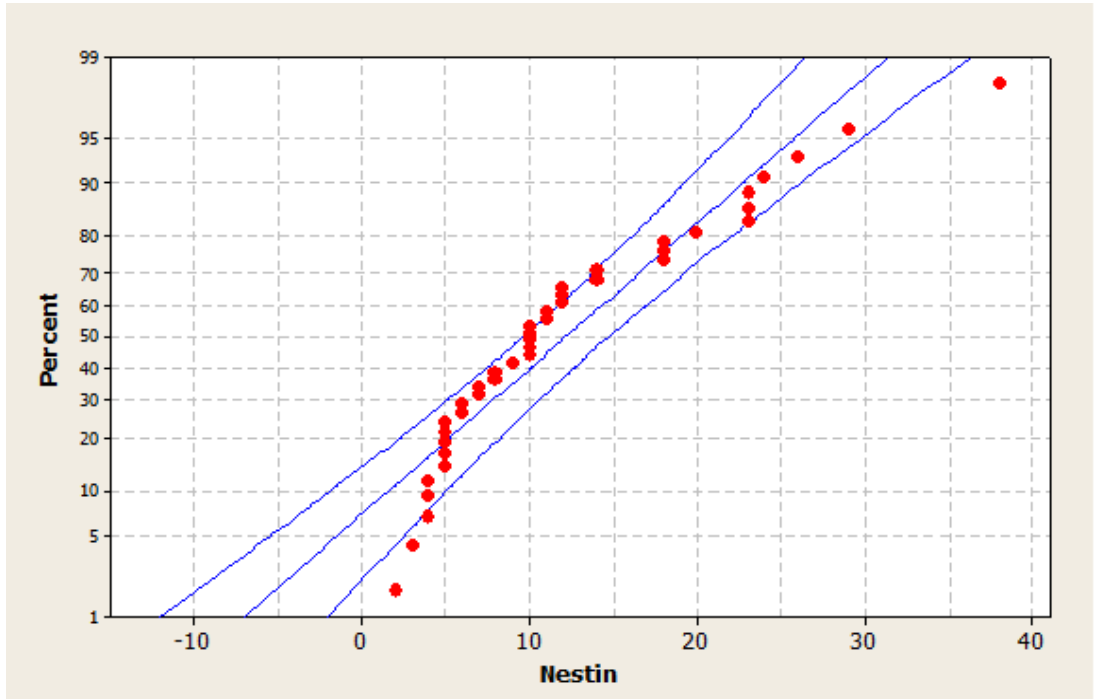

Figure 5. MVD and Nestin Expression

significantly associated with necrosis.

One of the important diagnostic factor in GBM is angiogenesis. So evaluation of tumor vascular density and anti-angiogenic therapies can play an important role in the patient's prognosis and recovery. Several studies have been conducted on micro vascular density (MVD) of tumorvessels using various vascular markers such as CD31 and CD34. These markers can stain both small and large vessels with the same intensity. In addition they are in both tumor and normal tissue vessels (Guadagno et al. 2016). Nestin is a class VI intermediate protein that expresses in malignant cells of GBM and can stain neoformed vessels. So it is a good marker for MVD evaluating (D'Alessio et al. 2016). A few studies have been performed on the MVD using Nestin and the angiogenesis process in GBM.

Chinnaiyan et al., (2008) and D'Alessio et al., (2016) have separately reported that Nestin expresses in the GBM stem cells and neoformed vessels endothelial. So in addition to the proliferation of tumor cells, it also stimulates the angiogenesis process.
In our research, the expression of Nestin in the vessels of malignant cells is related to the vascular proliferation in GBM. MVD in the areas with vascular proliferation is about 13.2. So Nestin is a sensitive marker for evaluating MVD.

Krupkova et al., (2006) have found that Nestin expression is related to the tumor grade and it is over expressed in high grade tumors. So it is a good candidate for GBM diagnosis (Veselska et al., 2006). In this study, there was no correlation between Nestin expression and necrosis or cellular atypia. Genetic mutations have a key role in forming GBMs. EGFR is an essential growth factor in epithelial tissues and abnormal signaling lead to the formation of epithelial malignity. It has been proposed that genetic mutation of EGFR gene is responsible for the pathogenesis of gliomas. Mechanisms for mutation of EGFR in malignity include structural rearrangements of the receptor, EGFR gene amplification, activating mutations in the EGFR kinase domain and overexpression of(EGF)-family ligands by tumor cells and/or surrounding stroma (Arif et al., 2015, Alamdari-Palangi et al., 2020). 
Schmidt et al., (2002) had a study on the glioblastoma samples in 2002 and indicated that EGFR gene amplification in less than $40 \%$ cases and overexpression in more than $60 \%$ cases are a remarkable point in GBM. These events are associated with increased invasion, adhesion, malignant cells proliferation and inducing of vascular proliferation (Simmons et al., 2001; Talasila et al., 2013).

In new GBM treatments approaches, it has been focused on the tyrosine kinase inhibition and growth factors-related pathways. Over activity of EGFR pathway is associated with resistance to the chemotherapy and radiotherapy. Therefore target therapy and targeted chemotherapy increase treatment effects (Arif et al., 2015).

In this study, expression of EGFR marker observed in $92.5 \%$ of cases in which $22.5 \%$ of cases have score 1 and $70 \%$ of cases have score 2 . But there isn't any correlation between EGFR expression and age, necrosis, vascular proliferation and cellular atypia.

Talasila et al., (2013) indicated that although severe mutation of EGFR in GBM is associated with nonvascular invasion, it can provide vascular proliferation on long term. In this study, we evaluated the rate of EGFR mutation in GBM and MVD, especially in neoformed vessels, using Nestin marker and the relation between angiogenesis and EGFR mutation. There is a positive correlation between MVD and EGFR mutation. It shows that mutations such as EGFR mutation, in addition to the tumor cells' proliferation can affect angiogenesis induction (Eskilsson et al., 2018).

In conclusion, we conclude that both EGFR mutation and inhibition of vascular proliferation are the remarkable therapeutic targets in GBM. So combination therapy against them can be effective in patients' prognosis. Further studies about the association between these targets are needed to find new diagnostic and therapeutic approaches for GBM.

\section{Acknowledgments}

The researcher wishes to express his appreciation to the full cooperation of the patients who participated in this study.

\section{Abbreviations}

WHO: world health organization

MVD: MicroVascular Density

EGFR: Epidermal Growth Factor Receptor

H\&E: Hematoxylinand Eosin

GBM: Glioblastoma Multiforme

VEGF: vascular endothelia growth factor

IHC: immunohistochemistry

\section{Funding}

The financial support for the current research (No. 96141) was provided by Research Deputy of Kashan University of Medical Sciences, Kashan, Iran.

\section{Availability of data and materials}

The dataset used in this study is available with the authors and can be made available upon request.
Nestin and EGFR in Patients with Glioblastoma Multiforme

\section{Authors' contributions}

All the authors participated in the study design. ZV and AHM collected and documented the data and assisted in preliminary data analysis. ZVand MMN wrote the initial draft. HHK participated in draft revision, data analysis and editing of the final draft.

\section{Consent for publication}

Not applicable.

\section{Ethics approval and consent to participate}

The study was approved by the ethics committee of the Kashan University of Medical Sciences with ID IR.KUMS.MEDNT.REC.1396.72. All procedures performed in studies involving human participants were in accordance with the ethical standards of the institutional and national research committee and with the 1964 Helsinki declaration and its later amendments.

\section{Declaration of competing interest}

The authors declared that they have no competing interests.

\section{References}

Alamdari-Palangi V, Amini R, Karami H (2020). MiRNA-7 enhances erlotinib sensitivity of glioblastoma cells by blocking the IRS-1 and IRS-2 expression. J Pharm Pharmacol, 5, doi: 10.1111/jphp.13226.

Arif SA, Pandith AR, Bhat A, et al (2015). EGFR and PTEN gene mutation status in glioblastoma patients and their prognostic impact on patient's survival. J Carcinog Mutagen, 6, 218.

Batash RN, Asna P, Schaffer N, et al (2017). Glioblastoma multiforme, diagnosis and treatment; recent literature review. Curr Med Chem, 24, 3002-9.

Chinnaiyan P, Wang M, Rojiani AM, et al (2008). The prognostic value of nestin expression in newly diagnosed glioblastoma: report from the Radiation Therapy Oncology Group. Radiat Oncol, 3, 32.

Cho J, Pastorino S, Zeng Q, et al (2011). Glioblastoma-derived Epidermal Growth Factor Receptor (EGFR) carboxylterminal deletion mutants are transforming and are sensitive to EGFR-directed therapies. Cancer Res, 71, 7587-96.

D'Alessio A, Proietti G, Lama G, et al (2016). Analysis of angiogenesis related factors in glioblastoma, peritumoral tissue and their derived cancer stem cells. Oncotarget, 7, 78541.

Dahlrot RH, Hansen S, Jensen SS, et al (2014). Clinical value of CD133 and nestin in patients with glioma: a populationbased study. Int J Clin Exp Patho, 7, 3739.

Eskilsson E, Røsland GV, Solecki G, et al (2018). EGFR heterogeneity and implications for therapeutic intervention in glioblastoma. Neuro Oncol, 20, 743-52.

Guadagno E, Borrelli G, Califano M, et al (2016). Immunohistochemical expression of stem cell markers CD44 and nestin in glioblastomas: Evaluation of their prognostic significance. Pathol Res Pract, 212, 825-32.

Hardee ME, Zagzag D (2012). Mechanisms of glioma-associated neovascularization. Am J Pathol, 181, 1126-41.

Ishiwata T, Teduka K, Yamamoto T, et al (2011). Neuroepithelial stem cell marker nestin regulates the migration, invasion and growth of human gliomas. Oncol Rep, 26, 91-9.

Jazayeri S B, Rahimi-Movaghar V, Shokraneh F, et al (2013). Epidemiology of primary CNS tumors in Iran: a sysematic. Asian Pac J Cancer Prev, 14, 3979-85.

Asian Pacific Journal of Cancer Prevention, Vol 21 
Jin X, Jin X, Jung J-E, et al (2013). Cell surface Nestin is a biomarker for glioma stem cells. Biochem Biophys Res Commun, 433, 496-501.

JR OK, Loja T, Zambo I, Veselska R (2010). Nestin expression in human tumors and tumor cell lines. Neoplasma, 57: 4.

Kitai R, Horita R, Sato K, et al (2010). Nestin expression in astrocytic tumors delineates tumor infiltration. Brain Tumor Patho, 27, 17-21.

Krupkova O, Loja T, Redova M, et al (2011). Analysis of nuclear nestin localization in cell lines derived from neurogenic tumors. Tumor Biol, 32, 631-9.

Lebelt A, Dziecioł J, Guzińska-Ustymowicz K, et al (2008). Angiogenesis in gliomas. Folia Histochem Cyto, 46, 69-72.

Liu F, Hon GC, Villa GR, et al (2015). EGFR mutation promotes glioblastoma through epigenome and transcription factor network remodeling. Mol Cell, 60, 307-18.

Loja T, Chlapek P, Kuglik P, et al (2009). Characterization of a GM7 glioblastoma cell line showing CD133 positivity and both cytoplasmic and nuclear localization of nestin. Oncol Rep, 21, 119-27.

Matsuda Y, Hagio M, Ishiwata T (2013). Nestin: a novel angiogenesis marker and possible target for tumor angiogenesis. World J Gastroenterol, 19, 42.

McNamara MG, Sahebjam S, Mason WP (2013). Emerging biomarkers in glioblastoma. Cancers, 5, 1103-19.

Moghaddam NA, Mahsuni P, Taheri D (2015). Evaluation of endoglin as an angiogenesis marker in glioblastoma. Iran J Pathol, 10, 89.

Mokrý J, Č́́žková D, Filip S, et al (2004). Nestin expression by newly formed human blood vessels. Stem Cells Dev, 13, 658-64.

Nagpal J, Jamoona A, Gulati ND, et al (2006). Revisiting the role of p53 in primary and secondary glioblastomas. Anticancer Res, 26, 4633-9.

Pallini R, Ricci-Vitiani L, Banna GL, et al (2008). Cancer stem cell analysis and clinical outcome in patients with glioblastoma multiforme. Clin Cancer Res, 14, 8205-12.

Sasmita AO, Wong YP, Ling APK (2018). Biomarkers and therapeutic advances in glioblastoma multiforme. Asia-Pac J Clin Onco, 14, 40-51.

Schmidt MC, Antweiler S, Urban N, et al (2002). Impact of genotype and morphology on the prognosis of glioblastoma. J Neuropathol Exp Neurol, 61, 321-8.

Shih AH, Holland EC (2006). Notch signaling enhances nestin expression in gliomas. Neoplasia (New York, NY), 8, 1072.

Sica G, Lama G, Anile C, et al (2011). Assessment of angiogenesis by CD105 and nestin expression in peritumor tissue of glioblastoma. Int J Oncol, 38, 41-9.

Simmons ML, Lamborn KR, Takahashi M, et al (2001). Analysis of complex relationships between age, p53, epidermal growth factor receptor, and survival in glioblastoma patients. Cancer Res, 61, 1122-8.

Smith SJ, Tilly H, Ward JH, et al (2012). CD105 (Endoglin) exerts prognostic effects via its role in the microvascular niche of paediatric high grade glioma. Acta Neuropathol, 124, 99-110.

Talasila KM, Soentgerath A, Euskirchen P, et al (2013). EGFR wild-type amplification and activation promote invasion and development of glioblastoma independent of angiogenesis. Acta Neuropathol, 125, 683-8.

Tena-Suck ML, Celis-Lopez MA, Collado-Ortiz MA, et al (2015). Glioblastoma multiforme and angiogenesis: A clinicopathological and immunohistochemistry approach. J Neurol Res, 5, 199-206.

Veselska R, Kuglik P, Cejpek P (2006). Nestin expression in the cell lines derived from glioblastoma multiforme. $B M C$ Cancer, 6, 32 .

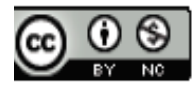

This work is licensed under a Creative Commons AttributionNon Commercial 4.0 International License. 